\title{
Articulación trabajo-familia y satisfacción laboral: El rol del compromiso afectivo
}

\section{Work-family interface and job satisfaction: The role of affective commitment}

\author{
Sebastián Gabinia,b,* \\ anstituto de Investigaciones, Facultad de Humanidades y Artes, Universidad Nacional de Rosario - CONICET, Argentina

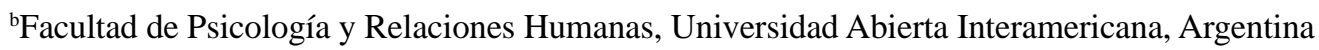

\section{Resumen}

Objetivo: el objetivo de la presente investigación fue analizar si alguno de los tipos de compromiso organizacional mediaba la relación entre la articulación trabajo-familia y la satisfacción laboral. Método: para ello, se diseñó un estudio empírico, cuantitativo y transversal, enmarcado en los lineamientos de la estrategia asociativa-explicativa. La medición de las variables de interés se llevó a cabo con una batería compuesta por instrumentos validados al contexto y que cuentan con adecuadas propiedades psicométricas. Se trabajó con una muestra no probabilística, por conveniencia, de 376 empleados (191 varones y 185 mujeres) de organizaciones del sector terciario, dada su importancia en el mundo actual del trabajo. Resultados: los resultados indicaron que el compromiso afectivo media parcialmente las relaciones entre las dimensiones del constructo articulación trabajo-familia (enriquecimiento y conflicto) y la satisfacción laboral. Este tipo de compromiso se erige como un constructo mediacional entre la satisfacción laboral y las posibilidades de articular las demandas de las distintas esferas vitales. Tal mecanismo puede ser explicado a la luz de la teoría del estrés de rol y de la teoría del intercambio social. Conclusiones: estos hallazgos pueden resultar de interés a la hora de diseñar prácticas de gerenciamiento amigables con la familia que posibiliten retener el talento humano y aumentar los lazos afectivos de los empleados con la organización.

Palabras clave: compromiso organizacional; articulación trabajofamilia; satisfacción laboral; mediación.

\section{Para citar este artículo:}

Gabini, S. (2020). Articulación trabajo-familia y satisfacción laboral: El rol del compromiso afectivo. Liberabit, 26(1), e352. https:// doi.org/10.24265/liberabit.2020.v26n1.06

\begin{abstract}
Objective: this research was to analyze if any of the types of organizational commitment mediated the relationship between work-family interface and job satisfaction. To that end, an empirical, quantitative and cross-sectional study was designed, framed within the guidelines of an associative-explanatory approach. Method: the study variables were measured using a set of validated instruments with adequate psychometric properties. The study population consisted of a non-probabilistic convenience sample of 376 workers (191 males and 185 females) from third-sector organizations, due to their importance in the current working world. Results: the results showed that affective commitment partially mediates the relationships between the dimensions work-family interface (enrichment and conflict) and job satisfaction. This type of commitment emerges as a mediational construct between job satisfaction and the possibilities of dealing with the demands from different vital spheres. Such mechanism may be explained through the role stress theory and the social exchange theory. Conclusions: these findings might be useful when it comes to designing familyfriendship policies in order to retain human talent and promote affective bonds between employees and organizations.
\end{abstract}

Keywords: organizational commitment; work-family interface; job satisfaction; mediation.

Este es un artículo Open Access bajo la licencia Creative Commons Atribución-NoComercial-CompartirIgual 4.0

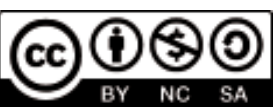




\section{Introducción}

Los cambios en relación a la vida laboral y familiar acontecidos en las últimas décadas, marcados por el crecimiento del número de mujeres trabajadoras, la emergencia de parejas donde ambos cónyuges se desempeñan en el mercado laboral externo y el incremento de familias monoparentales, han provocado alteraciones en los enfoques tradicionales de la organización del trabajo (Gabini, 2017; Timms et al., 2015). Estas modificaciones también han impactado los distintos sectores productivos al interior de la economía de un país. En efecto, el rubro servicios y comercios es uno de lo que más ha crecido en las últimas tres décadas $\mathrm{y}$, por lo tanto, se posiciona como una fuente de empleos que podría absorber a los trabajadores expulsados de los primeros sectores. En Argentina, particularmente, el sector terciario es el que más empleos proporciona a sus habitantes (más del $80 \%$ pertenece a empresas privadas) y genera más del $65 \%$ de su producto bruto interno (Oficina Económica y Comercial de España en Buenos Aires, 2018).

Ante esta nueva realidad, muchos empleados pertenecientes a este rubro se encuentran, día a día, sumergidos en el desafío de balancear sus roles familiares y laborales. Consecuentemente, la posibilidad de articular estas esferas vitales se ha transformado en un objeto de estudio primordial para los investigadores de la psicología organizacional, lo que ha generado un cuerpo sustancial de literatura específica sobre la temática (Allen, Johnson, Kiburz, \& Shockley, 2013).

En un primer momento, la investigación referida a esta problemática estuvo dominada por una perspectiva ligada al conflicto (Greenhaus \& Powell, 2006). De esta manera, el conflicto trabajo-familia (CTF) ha sido delimitado como la interferencia que ocurre entre los roles familiares y laborales, resultante de las presiones y demandas asociadas a cada dominio y que, además, puede llegar a tener efectos negativos sobre ambas esferas (Buonocore \& Russo, 2013). Posteriormente, algunos estudiosos de la temática
(Lingard, Francis, \& Turner, 2010) se han mostrado escépticos de la perspectiva del conflicto y han sugerido que las ventajas de participar simultáneamente en diversos roles son ser capaces de equilibrar las desventajas generadas entre estos dominios. A pesar de ello, las primeras investigaciones realizadas en este sentido se han visto obstaculizadas por la carencia de claridad conceptual y por la falta de un marco teórico general e integrador (McNall, Scott, \& Nicklin, 2015). Este desacuerdo en relación a la vertiente positiva de la interacción trabajo-familia, se ha traducido en la utilización de diversas designaciones para señalar al constructo tales como derrame positivo, mejoramiento o facilitación (Omar, Urteaga, \& Salessi, 2015). Con base en las dificultades provenientes de la falta de una denominación unívoca del fenómeno, Greenhaus y Powell (2006) propusieron el término enriquecimiento trabajo-familia (ETF) en referencia al grado en el que las experiencias vividas en un rol contribuyen a mejorar la calidad de vida en otros roles.

Sin embargo, el estudio aislado del conflicto o del enriquecimiento no ha provisto una base conceptual sólida para comprender la dinámica de la articulación entre ambas esferas. Por lo que desde hace más de una década, se ha comenzado a señalar que la naturaleza de la interacción trabajo-familia debe investigarse a partir de las combinaciones específicas de ambas perspectivas (Geurts et al., 2005; Michel \& Clark, 2009). Esta propuesta integradora ha generado la necesidad de definir los modos en que el aspecto positivo y el negativo se relacionan, vale decir, si se trata de constructos isomorficos (aspectos opuestos de un continuum con los mismos antecedentes y consecuencias) u ortogonales (dimensiones distintas con antecedentes y consecuencias particulares). En términos generales, se observan mayores acuerdos sobre la ortogonalidad de ambos constructos (Michel \& Clark, 2009), así como sobre la necesidad de estudiarlos conjuntamente para lograr un abordaje holístico de la interacción trabajo-familia (Padhi \& Pattnaik, 2013; Rantanen, Kinnunen, Mauno, \& Tement, 2013; Romeo, Berger, Montserrat, \& Ramos, 2014). 
La revisión de la bibliografía muestra que existen mayores evidencias sobre los antecedentes que sobre las consecuencias de ambos constructos. En este sentido, como antecedentes del ETF se destacan las políticas amigables con la familia (Crain \& Hammer, 2013), la flexibilidad de horario y de lugar (Jijena-Michel \& Jijena-Michel, 2015; Rastogi, Rangnekar, \& Rastogi, 2016), así como las condiciones de trabajo que contemplen seguridad laboral, autonomía y prestigio (Cooklin et al., 2015). En tanto que como antecedentes del CTF se han indicado, por un lado, características laborales tales como ambigüedad de rol, sobrecarga de rol, demandas de tiempo, inseguridad laboral, modalidad de contratación y, por el otro, características familiares como el números de hijos o personas a cargo, la falta de apoyo marital o clima familiar (Cooklin et al., 2015; Mauno et al., 2015).

En relación con las consecuencias, mientras que las ligadas al CTF han sido ampliamente exploradas (Scott, Ingram, Zagenczyk, \& Shoss, 2015), las relacionadas con el ETF no han arribado al mismo nivel de sistematización (Padhi \& Pattnaik, 2013). Particularmente, se ha señalado que el incremento en los niveles de CTF contribuye a aumentar las tensiones psicológicas (Brummelhuis \& Bakker, 2012), el cansancio emocional (Leineweber, Baltzer, Hanson, \& Westerlund, 2013) y el burnout (Bagherzadeh et al., 2016). En términos generales, existe consenso en que el CTF predice negativamente el bienestar (Mauno et al., 2015) y la salud del empleado (Leineweber et al., 2013). En contraposición, se ha indicado que el ETF tiene un efecto directo sobre la reducción del cansancio laboral y las intenciones de renunciar (Mauno et al., 2015), el bienestar cultural del lugar de trabajo (Brougham, Haar, \& Roche, 2015), la generación de sentimientos positivos hacia el trabajo (Wayne, Randel, \& Stevens, 2006) y el compromiso organizacional (McNall et al., 2015; Timms et al., 2015).

A pesar de tales evidencias, hasta la fecha no se ha evaluado de manera conjunta la influencia del CTF y ETF sobre algunos resultados laborales significativos, tales como el grado de satisfacción laboral del empleado. Ni tampoco se ha explorado si estas posibles relaciones (directas o bivariadas) podrían estar influenciadas por terceras variables que medien o moderen su intensidad y dirección. El presente trabajo se enmarcó, justamente, en esta perspectiva conocida como «de segunda generación» (Hopwood, 2007), orientada a identificar terceras variables que faciliten la asociación entre variables antecedentes y consecuentes. Concretamente, el objetivo del presente estudio fue analizar si alguno de los tipos de compromiso organizacional desempeña el rol de mediador (total o parcial) entre la articulación trabajo-familia (ETF y CTF) y la satisfacción laboral de los trabajadores.

\section{Articulación trabajo-familia y satisfacción laboral}

Desde su irrupción en el ámbito académico, la satisfacción laboral ha sido objeto de diversas conceptualizaciones. Estas incluyen desde su consideración como un sentimiento positivo, a su definición en términos de la favorabilidad (o desfavorabilidad) con la que los empleados evalúan su trabajo (Salessi, 2014). En los últimos años, ha comenzado a cobrar fuerza la alternativa de concebirla como un conjunto de respuestas cognitivas y afectivas a la situación laboral, con importantes implicancias en el comportamiento de los empleados y en los resultados organizacionales (Judge \& Kammeyer-Mueller, 2012). A nivel individual, los trabajadores con índices elevados de satisfacción laboral suelen experimentar un mayor grado de autoestima, felicidad y bienestar. Mientras que, a nivel institucional, las organizaciones pueden verse recompensadas por rendimientos destacados, que se traducen en incrementos de la productividad y el compromiso, así como en menores intenciones de renunciar por parte de sus miembros (Salessi \& Omar, 2017).

En lo que hace a su relación con la articulación trabajo-familia, si bien la mayor parte de los estudios ha focalizado en el rol de variables contextuales ligadas al clima organizacional y al diseño del puesto, alguna 
evidencia indica que la posibilidad de balancear los roles familiares y laborales influye sobre la satisfacción del empleado. Al respecto, se ha encontrado (AlAzzam, AbuAlRub, \& Nazzal, 2017; Mauno \& Ruokolainen, 2017) que el CTF predice negativamente la satisfacción laboral en diferentes países, aunque con mayor impacto en aquellos donde prima una cultura individualista (Haar, Russo, Suñe, \& Ollier-Malaterre, 2014). Por otra parte, resultados empíricos recientes (Brougham et al., 2015; McNall et al., 2015) coinciden en afirmar que el ETF se relaciona con un mayor grado de satisfacción laboral. Este doble aspecto de la articulación trabajo-familia y su relación con la satisfacción puede encontrar fundamento en el modelo de las demandas y recursos laborales. Ambos se presentan como los factores desencadenantes de los procesos de deterioro de la salud (energético) y procesos motivacionales. Entonces, mientras que las demandas laborales son predictoras de variables como el agotamiento, los recursos son antecedentes de la satisfacción en el trabajo y el engagement (Bakker \& Demerouti, 2013).

En base a estos antecedentes se tienen las siguientes hipótesis:

- Hipótesis 1: El CTF se relacionará negativamente con la satisfacción laboral.

- Hipótesis 2: El ETF se relacionará positivamente con la satisfacción laboral.

\section{Los tipos de compromiso organizacional como variables mediadoras}

En la actualidad, el modelo de compromiso organizacional propuesto por Allen y Meyer (1990) ha alcanzado un importante grado de consenso entre los especialistas en la temática (Albdour \& Altarawneh, 2014; Liu \& Wang, 2013; Muneer, Iqbal, Khan, \& Long, 2014). Desde esta perspectiva, el constructo ha sido definido como el grado con que las personas se identifican con sus organizaciones, se encuentran consustanciadas con las mismas, y están dispuestas a continuar trabajando en ellas. El modelo en cuestión distingue entre tres tipos de compromiso en función de los motivos por los que un trabajador desea continuar formando parte de su organización: compromiso afectivo (lazos emocionales que unen al individuo con la organización), compromiso calculativo (toma de conciencia por parte del empleado de los altos costos que generaría el hecho de dejar la organización) y compromiso normativo (obligación del empleado de permanecer en la organización no solo porque está contratado, sino porque cree que esto es lo correcto). En relación a ello, se ha indicado que es probable que los empleados experimenten diferencialmente las tres formas de compromiso. Un empleado, por ejemplo, puede sentir tanto un fuerte deseo (afectivo) y necesidad de permanecer (calculativo), pero poca obligación (normativo) de hacerlo (Allen \& Meyer, 1990).

El hipotético rol de la tercera variable se fundamenta en las conclusiones de algunos estudiosos recientes que subrayan la función mediadora del compromiso afectivo (Fazio, Gong, Sims, \& Yurova, 2017; Wong \& Wong, 2017), normativo (Al Momani, 2017) o calculativo (Kaur \& Soch, 2018), entre los sentimientos generados por articular las demandas del trabajo con las de la familia (como el apoyo organizacional percibido o la calidad de la vida laboral) y las actitudes vinculadas con el trabajo (tales como las intenciones de renuncia o el rendimiento). Estos antecedentes, proponen un rol mediador dado que se han centrado en la explicación de los procesos y mecanismos que producen determinados efectos, es decir, cómo se explica la relación entre una variable independiente y una variable dependiente. Para, de esta manera, buscar explicaciones (teóricas) que ayuden a entender los procesos que operan en la realidad.

Este mecanismo puede ser explicado a la luz de la teoría del intercambio social, cuya presunción general es que los empleados pueden establecer, desde su rol laboral, diferentes acuerdos sociales que tienen implicancias en sus modos de comportarse. De esta manera, es probable que los trabajadores tiendan a intercambiar su compromiso con la organización por el apoyo de su empleador, muchas veces ligado a 
determinadas políticas amigables con la familia que conllevan la posibilidad de balancear ambos roles (Cropanzano, Anthony, Daniels, \& Hall, 2017). Sumado a ello, la teoría del estrés de rol sostiene que la necesidad de cumplimentar con las demandas generadas a partir de la participación en múltiples roles tiende a generar estrés. Sin embargo, los empleados comprometidos generan sentimientos de estabilidad y de seguridad para con la empresa que le permiten hacer frente a estos estresores (Buonocore \& Russo, 2013).

Particularmente, investigaciones empíricas han indicado que el compromiso organizacional media la relación entre la satisfacción laboral y los comportamientos de ciudadanía organizacional (Chhabra \& Mohanty, 2016), las condiciones laborales, el salario y la seguridad laboral (Sangadji, 2014). Sin embargo, hasta el momento no se tiene conocimiento de estudios que hayan indagado el rol mediador de alguno de los tipos de compromiso afectivo en la relación entre la articulación trabajo-familia y la satisfacción laboral. Por ello, se conjetura lo siguiente:

- Hipótesis 3: Al menos uno de los tipos de compromiso organizacional mediará las relaciones entre el CTF y la satisfacción laboral.

- Hipótesis 4: Al menos uno de los tipos de compromiso organizacional mediará las relaciones entre el ETF y la satisfacción laboral.

\section{Método}

La presente investigación corresponde a un estudio empírico de corte cuantitativo y transversal. El mismo se enmarca en los lineamientos de la estrategia asociativa-explicativa (Ato, López, \& Benavente, 2013), ya que su finalidad fue explorar la relación funcional existente entre variables.

\section{Participantes y procedimiento}

Se trabajó con una muestra por conveniencia no probabilística de 376 empleados (191 varones y 185 mujeres) del sector terciario que mantenían interacciones interpersonales frecuentes con clientes. El mayor porcentaje de los participantes tenía edades comprendidas entre los 21-30 años (37.9\%) y los 3140 años (28.7\%). Porcentajes menores se ubicaron en los rangos etarios comprendidos entre los 18-20 (13.6\%) y entre $41-50$ años (11.7\%), en tanto que el rango de los mayores de 50 años (8.1\%) fue el menos representado.

Para la selección de la muestra se tomó contacto con diversas organizaciones públicas y privadas que ofrecieran distintos tipos de servicios, localizadas en la ciudad de Rosario (provincia de Santa Fe, Argentina) invitándolas a participar del estudio. Con aquellas que aceptaron colaborar se pautaron días y horarios para concretar la recolección de los datos. Para ello, se acordó hacerles llegar una determinada cantidad de protocolos para que los empleados pudieran responderlos en sus momentos libres, de modo de no generar interrupciones durante la jornada laboral. Las cédulas respondidas fueron retiradas una semana después de la recepción. Para cumplimentar con el número de casos esperados (más de 300), se confeccionaron y entregaron 750 cédulas de recolección de datos. Del total entregados, cerca de $60 \%$ fueron recuperados; sin embargo, un buen número de protocolos (cerca de un 10\%) debieron ser descartados por encontrarse incompletos o presentar patrones de respuesta que denotaban una clara falta de compromiso (por ejemplo, seleccionar la misma opción de respuesta para todos los reactivos).

\section{Instrumentos}

Variables personales. A cada empleado se le solicitó información acerca de su género, edad y rubro de la empresa.

Satisfacción laboral. Se utilizó la Escala Satisfacción Laboral Genérica de MacDonald y MacIntyre (1997), adaptada por Salessi y Omar (2016). Las propiedades psicométricas de esta versión fueron evaluadas a través de análisis factoriales (exploratorios y confirmatorios) que indicaron una estructura unidimensional, correlaciones 
entre los puntajes de satisfacción laboral y compromiso $(r=.81 ; p<.01)$, confianza $(r=.84 ; p$ $<.01)$ y cinismo organizacional $(r=-.70 ; p<.01)$ para medir su validez convergente, y el método de consistencia interna $(\alpha=.87)$ para estimar su confiabilidad. El instrumento, integrado por 7 ítems (e.g., «En mi trabajo puedo aplicar todas mis capacidades y habilidades.»), proporciona una estimación global del grado de satisfacción laboral. Cada reactivo es evaluado según una escala tipo Likert con 5 opciones de respuesta ( 1 = totalmente en desacuerdo; 5 = totalmente de acuerdo). A mayor puntaje, mayor satisfacción laboral.

Compromiso organizacional. Fue evaluado mediante la adaptación argentina (Omar \& Urteaga, 2008) de las escalas de Compromiso Organizacional de Allen y Meyer (1990) para valorar el modelo de tres componentes: afectivo (e.g., «Esta empresa tiene mucho significado para mí.»), normativo (e.g., «Siento que tengo una gran deuda hacia mi empresa.») y calculativo (e.g., «Siento que tengo pocas opciones de trabajo como para considerar dejar mi organización.»). Se trata de escalas independientes que miden constructos distinguibles entre ellos. Con base en ello, innumerables investigaciones han elegido medir el modelo completo o alguno de sus tres componentes (Meyer, Stanley, Herscovitch, \& Topolnytsky, 2002). La versión validada se encuentra conformada por seis ítems para cada una, con formato Likert de 5 puntos (variando desde $1=$ totalmente en desacuerdo a 5 = totalmente de acuerdo). Los índices de consistencia interna (alfa de Cronbach) fueron de .82 para el compromiso afectivo, de .73 para el normativo y de .76 para el calculativo.

Articulación trabajo-familia. Se exploró mediante la adaptación argentina (Gabini, 2017) del Cuestionario de Interacción Trabajo-Familia (SWING; Geurts et al., 2005), integrada por ocho ítems con formato Likert de 5 puntos (variando desde $1=$ nunca a 5 = siempre). Esta escala explora tanto los recursos ganados en las esferas familiares y laborales, denominado enriquecimiento trabajo-familia, a través de cuatro ítems (e.g., «Tengo más autoconfianza en el trabajo porque mi vida en casa está bien organizada.»); así como el vínculo negativo entre ambos dominios, conflicto trabajo-familia, a partir de cuatro ítems (e.g., «Me resulta difícil concentrarme en mi trabajo porque estoy preocupado por asuntos domésticos.»). Para la adaptación y validación de la escala, se ejecutaron análisis factoriales exploratorios y confirmatorios, los que indicaron una estructura bifactorial con adecuada consistencia (ETF: $\alpha=.76$ y CTF: $\alpha=.74$ ), confiabilidad compuesta (mayores a .70) y validez convergente (mayor a .50).

\section{Consideraciones éticas}

El contacto con los empleados y la aplicación de la cédula de recolección de datos se llevó a cabo en los lugares habituales de trabajo, previa solicitud de autorización a las autoridades organizacionales. La participación fue voluntaria y la totalidad de los participantes firmaron un protocolo de consentimiento informado. Se garantizó el anonimato y la confidencialidad de la información suministrada. Durante todo el proceso se tomaron en consideración todos los recaudos éticos vinculados con la investigación con seres humanos establecidos por la Asociación Americana de Psicología (2017) y las recomendaciones del CONICET (2006) para las investigaciones en las ciencias sociales y humanas.

\section{Análisis de datos}

Se examinó la matriz de datos con miras a detectar valores perdidos y/o atípicos. Se calcularon los índices descriptivos (medias y desvíos típicos) y los coeficientes de correlación entre los constructos en estudio con el objeto de verificar qué aspecto o aspectos del compromiso organizacional retener para los posteriores análisis. A continuación, se analizó la distribución conjunta de las variables a través del cómputo del coeficiente de curtosis multivariada estandarizado (Bentler, 2006) y se ejecutó un análisis factorial confirmatorio (AFC) para determinar el ajuste 
del modelo de medida utilizado. En este caso, un modelo conformado por los factores ETF, CTF, dimensión del compromiso organizacional retenida y satisfacción laboral; sus respectivos ítems como indicadores observables y sus respectivos términos de error. La confiabilidad se examinó a partir del índice de confiabilidad compuesta (CR, Composite Reliability), recomendado cuando se tienen en cuenta las interacciones entre dos o más constructos. La validez convergente se determinó a través de la varianza media extraída (AVE, Average Variance Extracted). La validez discriminante se analizó con base en la raíz cuadrada del AVE (Fornell \& Larcker, 1981). La presencia de potenciales sesgos debidos al método común se evaluó a partir de la prueba de un solo factor de Harman (Podsakoff, MacKenzie, \& Podsakoff, 2012). Complementariamente, se realizaron pruebas de diferencias de medias ( $t$ de Student) para el género y análisis de varianza (ANOVA de un factor) para la edad de los participantes. Tal procedimiento se realizó a fin de detectar posibles diferencias significativas que pudieran ameritar incluir a estas características sociodemográficas como variables control.

La verificación empírica de las hipótesis propuestas se realizó mediante la modelización con ecuaciones estructurales, a partir de la estrategia de modelos rivales (Bentler, 2006) que permite contrastar dos modelos distintos: (a) un modelo de efectos indirectos o de mediación total (modelo A) y (b) un modelo de efectos indirectos y directos o de mediación parcial (modelo B). En el modelo A, el ETF y el CTF se desempeñaron como variables independientes con efectos indirectos sobre la satisfacción laboral a través de la dimensión del compromiso organizacional retenida. En el modelo $B$, se añadieron efectos directos residuales por parte de las variables antecedentes sobre la satisfacción laboral. Para la estimación de los modelos se empleó el método de máxima verosimilitud con la corrección robusta de Satorra-Bentler (S-B), recomendado cuando los datos provienen de escalas tipo Likert (Bentler, 2006). Para evaluar la bondad de ajuste se analizó que la razón entre índice chi-cuadrado corregido no fuera significativo y los grados de libertad
$\left(\mathrm{S}-\mathrm{B} \chi^{2} / \mathrm{gl}\right)$ fuera inferior a 4; que el índice de bondad de ajuste (GFI, Goodness of Fit Index) y el índice de ajuste comparativo (CFI, Comparative Fit Index) alcanzaran valores iguales o superiores a .90 , y que el valor del error cuadrático medio de aproximación (RMSEA, Root Mean Square Error of Approximation) fuera inferior a .05. Adicionalmente, se analizó el índice esperado de validación cruzada (ECVI, Expected Cross-Validation Index) y el criterio consistente de información de Akaike (CAIC, Consistent Akaike Information Criterion), considerando que el modelo más adecuado sería el que presente valores comparativamente más pequeños. Complementariamente, se computó la prueba de diferencias entre los estadísticos chi-cuadrado ( $\left.\mathrm{S}-\mathrm{B} \chi^{2}\right)$ y se ejecutó un análisis con bootstrapping para determinar la significación estadística de los efectos totales, directos e indirectos. Con tal propósito, se seleccionaron 5000 muestras al azar del conjunto de datos y se estimaron los intervalos de confianza al 95\%. Comparada con los métodos tradicionales (Baron \& Kenny, 1986; Mackinnon, Lockwood, Hoffman, West, Sheets, 2002; Sobel, 1982), esta alternativa es más flexible y fácil de utilizar. Los análisis fueron ejecutados con los softwares SPSS (versión 23) y EQS (versión 6.1).

\section{Resultados}

\section{Análisis preliminares}

Sobre las 376 cédulas se efectuó una exploración preliminar para identificar la presencia de valores perdidos y/o observaciones atípicas que pudieran afectar los resultados del estudio. Esta inspección demostró que no existían valores perdidos que pudieran disminuir la eficacia de las pruebas estadísticas. Tampoco se encontraron observaciones extremas univariadas (Tabachnick \& Fidell, 2013) o multivariadas (Hair, Black, Babin, Anderson, \& Tatham, 2010). En definitiva, la totalidad de los casos fueron considerados válidos para continuar con los análisis posteriores. 
En la Tabla 1 se presentan los estadísticos descriptivos y los coeficientes de correlación bivariada para la muestra total. Como se desprende de la misma, los tres tipos de compromiso organizacional se correlacionaron con la satisfacción laboral y el ETF, siendo el compromiso afectivo el que presentó las asociaciones más fuertes. Por otra parte, el CTF se correlacionó negativa y significativamente con el compromiso afectivo $(r=-$ $.14 ; p<.01)$ y no con los otros compromisos. A raíz de tales resultados se optó por retener solo al componente afectivo para los análisis posteriores, dado que era la única dimensión constructo que se vinculaba con las demás variables de interés.

Tabla 1

Medias, desvíos típicos y correlaciones entre los factores del compromiso organizacional, la satisfacción laboral, el CTF y el ETF

\begin{tabular}{|c|c|c|c|c|c|c|c|c|}
\hline & Media & DT & 1 & 2 & 3 & 4 & 5 & 6 \\
\hline 1. $\mathrm{CN}$ & 3.07 & .79 & - & .42 & .68 & .46 & -.00 & .34 \\
\hline 2. CC & 3.12 & .67 & & - & .27 & .12 & .10 & .15 \\
\hline 3. CA & 3.26 & .97 & & & - & .63 & -.14 & .35 \\
\hline 4. SL & 3.85 & .66 & & & & - & -.29 & .40 \\
\hline 5. CTF & 2.44 & .82 & & & & & - & -.19 \\
\hline 6. ETF & 3.59 & .75 & & & & & & - \\
\hline
\end{tabular}

Nota: Negrita $=p<.01 ;$ cursiva $=p<.05$

El coeficiente de curtosis multivariante estandarizado fue de 28.31, ubicándose muy por encima de los límites estipulados $(-3 ; 3)$ para asumir multinormalidad (Bentler, 2006). Con base en dicho resultado, se optó por el uso de estimadores robustos para el cálculo de los parámetros, tanto del modelo de medida como del modelo estructural. El modelo de medida quedó conformado por cuatro variables correlacionadas: ETF, CTF, compromiso afectivo y satisfacción laboral. A cada variable latente se le sumaron sus respectivos indicadores y los correspondientes errores de medida. El modelo de medida inicial no logró un ajuste totalmente satisfactorio
$\left(\mathrm{S}-\mathrm{B} \chi^{2} / \mathrm{gl}=9.36, \mathrm{GFI}=.86, \mathrm{CFI}=.85, \mathrm{RMSEA}=\right.$ $.06[\mathrm{IC}=.05 ; .06])$. Sin embargo, a partir de la eliminación de tres indicadores que no se vincularon significativamente con la variable correspondiente (ítem 4 correspondiente a satisfacción laboral, ítem 3 correspondiente a ETF e ítem 4 correspondiente a CTF) se consiguió un ajuste más adecuado a los datos empíricos $\left(\mathrm{S}-\mathrm{B} \chi^{2} / \mathrm{gl}=1.87\right.$, GFI $=.91$, CFI $=.92$, RMSEA = .04 [IC = .03; .05]). La Tabla 2 presenta los resultados derivados de las pruebas de confiabilidad y validez del modelo de medida. En todos los casos, los índices de confiabilidad compuesta superaron el mínimo de .70, el índice AVE para cada constructo fue

Tabla 2

Confiabilidad y validez correspondientes a las variables en estudio

\begin{tabular}{cccc}
\hline & CR & AVE & Raíz cuadrada de AVE \\
\hline CA & .88 & .55 & .74 \\
SL & .86 & .51 & .71 \\
CTF & .76 & .52 & .72 \\
ETF & .78 & .55 & .74 \\
\hline
\end{tabular}

Nota: $\mathrm{CR}=$ Composite Reliability; AVE = Average Variance Extracted. 
mayor a .50, y la raíz cuadrada del mismo fue superior que la correlación entre ese constructo y todos los demás (Fornell \& Larcker, 1981). Por su parte, la prueba del factor único de Harman indicó que los datos no se encontraban sesgados desde el momento que la varianza explicada por un solo factor era del $27.40 \%$.

En lo que hace a las diferencias de medias en función de las variables sociodemográficas, la prueba $t$ de Student para la variable sexo no arrojó resultados significativos. En cuanto a la edad de los trabajadores, solo se encontró una exigua diferencia en relación al compromiso afectivo entre los empleados mayores de 50 años y aquellos que tenían entre 19 y 30 años de edad. Dado que solo se encontró una diferencia entre la totalidad de las comparaciones de las medias obtenidas por los cinco rangos etarios propuestos para cada uno de los constructos de interés (CTF, ETF, compromiso afectivo y satisfacción), se optó por no incluir las variables sociodemográficas (control) en los análisis posteriores.

\section{Verificación empírica}

La modelización con ecuaciones estructurales permitió conocer la magnitud y dirección de los coeficientes de regresión entre las relaciones estipuladas. En este sentido, el ETF se relacionó positivamente con la satisfacción laboral $(\beta=.46, p$ $<.001)$ y el CTF lo hizo negativamente $(\beta=-.12, p$ $<.001)$. Estos resultados brindan apoyo empírico a las hipótesis 1 y 2. De manera similar, el ETF se vinculó positivamente con el compromiso afectivo ( $\beta=.53, p$
$<.001$ ), en tanto que el CTF se relacionó negativamente con este tipo de compromiso $(\beta=-.12$, $p<.05)$. Por su parte, el compromiso afectivo se asoció de manera positiva a la satisfacción laboral $(\beta=.25$, $p<.001$ ). De este modo, se pudo demostrar que se cumplían las premisas que rigen el mecanismo de mediación.

La comparación de los índices de ajuste indicó que el modelo de mediación parcial se ajustaba mejor a los datos empíricos $\left(\mathrm{S}-\mathrm{B} \chi^{2}=5.14\right.$; $\mathrm{S}-\mathrm{B} \chi^{2} / \mathrm{gl}=5.14$; GFI $=.92 ; \mathrm{CFI}=.93$; RMSEA $=.04$; ECVI = 1.03; CAIC $=54.52)$ que el modelo de mediación total $\left(\mathrm{S}-\mathrm{B} \chi^{2}=\right.$ 25.94; $\mathrm{S}-\mathrm{B} \chi^{2} / \mathrm{gl}=12.97 ; \mathrm{GFI}=.77$; $\mathrm{CFI}=.73$; RMSEA = .07; ECVI = 1.12; CAIC $=245.59$ ).

Posteriormente, el análisis de bootstrapping permitió ratificar tales resultados, así como determinar la significancia estadística de los efectos entre las variables. De la Tabla 3 se desprende que los efectos indirectos de las dimensiones de la articulación trabajofamilia sobre la satisfacción laboral a través del compromiso afectivo son estadísticamente significativos, lo que demuestra la existencia de mediación. Sin embargo, a pesar de la reducción de la magnitud de los valores absolutos (efectos totales) en presencia del compromiso afectivo (efectos directos), el hecho de que las variables independientes sigan teniendo efectos significativos sobre la satisfacción laboral indica un tipo de mediación parcial. De este modo, se confirma empíricamente la hipótesis 3 y 4 en tanto un tipo de compromiso organizacional (afectivo) cumplía el rol de tercera variable propuesto.

Tabla 3

Coeficientes estandarizados e intervalos de confianza correspondientes a los efectos indirectos y directos del ETF y del CTF sobre la SL

\begin{tabular}{ccccccc}
\hline Relación & \multicolumn{2}{c}{ Efectos totales } & \multicolumn{2}{c}{ Efectos directos } & \multicolumn{2}{c}{ Efectos indirectos } \\
\hline & $\beta$ & IC al 95\% & $\beta$ & IC al 95\% & $\beta$ & IC al 95\% \\
\hline ETF=>SL & $\mathbf{. 6 0}$ & $.54 ; .66$ &. $\mathbf{4 6}$ & $.39 ; .54$ &. $\mathbf{1 3}$ & $.09 ; .18$ \\
CTF=>SL & -.15 & $-.23 ;-.08$ & -.12 & $-.20 ;-.05$ & -.03 & $-.05 ;-.01$ \\
\hline
\end{tabular}

Nota: negrita $=p<.001 ;$ cursiva $=p<.01 ;$ ETF = enriquecimiento trabajo-familia; CTF = conflicto trabajo-familia; $\mathrm{SL}=$ satisfacción laboral; IC = intervalo de confianza. 
La Figura 1 resume los coeficientes de regresión estandari-zados y las proporciones de varianzas explicadas $\left(\mathrm{R}^{2}\right)$ para la variable dependiente $\mathrm{y}$ mediadora. En este caso el modelo verificado explica cerca de un $50 \%$ de la varianza de la satisfacción laboral.

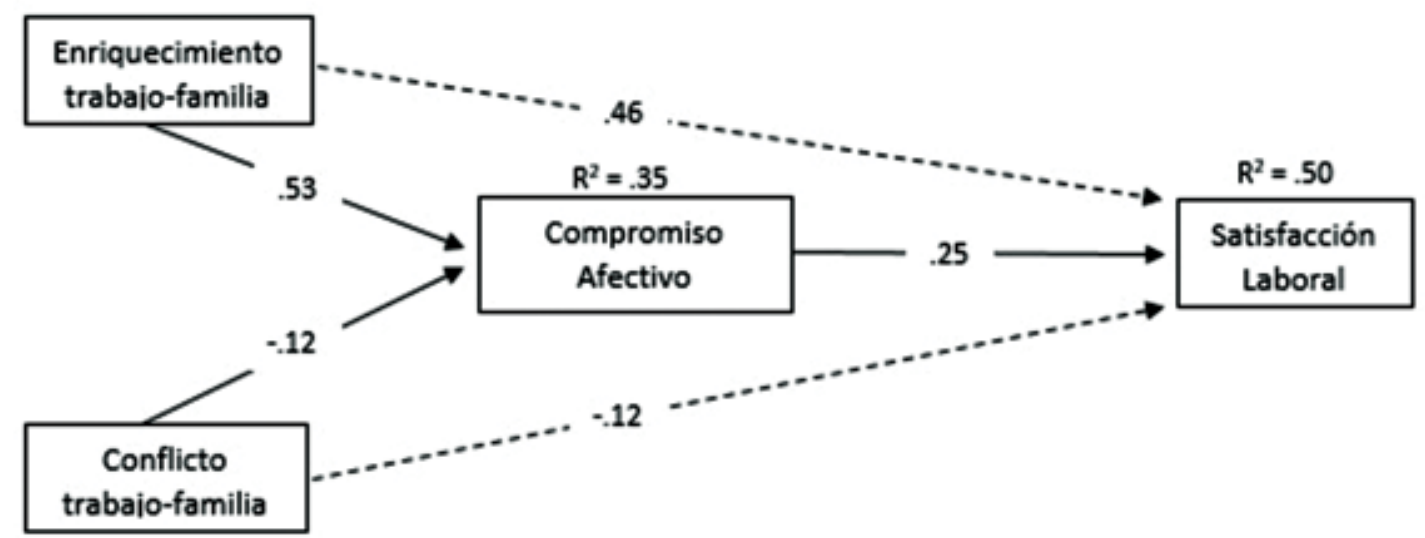

Figura 1. Coeficientes estandarizados y proporción de varianza explicada correspondiente al modelo de mediación parcial. Las líneas cortadas representan los efectos directos residuales; mientas que las líneas continuas, los efectos indirectos.

\section{Discusión}

El objetivo de la presente investigación fue analizar si alguno de los tipos de compromiso organizacional mediaba la relación entre las dos dimensiones de la articulación trabajo familia (ETF y CTF) y la satisfacción laboral del empleado. En función a la inspección de los análisis preliminares, se escogió el compromiso afectivo dado que era el único que se correlacionaba con las otras variables de interés. Esta elección se encuentra en línea con lo expuesto por Simintiras, Watkins, Ifie y Georgakas (2012) al señalar que este aspecto es una medida más efectiva del compromiso organizacional desde el momento que ha demostrado tener mayores efectos sobre los resultados de los trabajadores que los otros dos tipos de compromiso. Posteriormente, se elaboraron dos modelos teóricos rivales en los que el compromiso afectivo recibió los roles de mediador total y parcial. La modelización mediante ecuaciones estructurales de los mismos permitió concluir que el modelo de mediación parcial era el que mejor se ajustaba a los datos empíricos. A partir de los resultados obtenidos se pudo confirmar que los lazos emocionales del empleado con su organización establecen un puente entre los demás constructos de interés. Sin embargo, el hecho de que la mediación sea parcial podría indicar la presencia de otras variables mediadoras entre la articulación trabajo-familia y la satisfacción laboral.

El modelo explicativo seleccionado, además, permitió establecer otras relaciones significativas entre las variables de interés. En este sentido, el compromiso afectivo se vinculó simultáneamente con el CTF y el ETF. Las asociaciones negativas entre este tipo de compromiso y la faceta negativa de la articulación trabajo-familia han sido ampliamente documentadas en la bibliografía previa (Buonocore \& Russo, 2013). Por su parte, si bien la relación entre el ETF y el compromiso afectivo no ha arribado al mismo nivel de sistematización, algunas investigaciones recientes (Marques, Chambel, \& Pinto, 2015; Rahman, Sa'adah, Amin, Mahadi, \& Ismail, 2017) han señalado una vinculación positiva entre ambos constructos.

La satisfacción laboral presentó asociaciones significativas con las dos dimensiones de la articulación trabajo-familia. Algunos estudios 
empíricos actuales (AlAzzam et al., 2017; Mauno \& Ruokolainen, 2017) han corroborado que mientras el CTF predice negativamente la satisfacción laboral, el ETF lo hace de manera positiva (Brougham et al., 2015; McNall et al., 2015). Las razones de estos efectos son que el trabajo exige esfuerzo y consume recursos energéticos (demandas), mientras que los recursos laborales satisfacen necesidades humanas básicas, como autonomía y autoeficacia, y permiten la persecución de objetivos concordantes con uno mismo. De hecho, estas personas se encuentran intrínsecamente motivadas a perseguir tales objetivos y, por tanto, experimentan mayor satisfacción (Bakker \& Demerouti, 2013). Sin embargo, a la fecha no existen suficientes evidencias que valoren el impacto simultáneo de ambas facetas de la interacción trabajo-familia sobre este resultado laboral del modo que lo hace la presente investigación. Esta perspectiva se torna imprescindible para futuras investigaciones dado que los individuos experimentan ambos fenómenos de manera simultánea (Padhi \& Pattnaik, 2013). Por lo tanto, no debieran ser estudiados como compartimentos estancos sin interacción o influencia entre ellos, ya que ninguno de estos mecanismos aislados provee una base conceptual sólida para comprender la dinámica de la articulación entre ambas esferas (Frone, 2003).

Por su parte, el rol que cumple el compromiso afectivo en este modelo se condice con los resultados provenientes de estudios más recientes que han destacado a este tipo de compromiso como un constructo mediacional entre la satisfacción laboral y algunas posibilidades de articular las demandas de las distintas esferas vitales, tales como las condiciones laborales (Yousef, 2017), las prácticas de gerenciamiento (Leggat, Karimi, \& Bartram, 2017) o los comportamientos cívicos al interior de las organizaciones (Chhabra \& Mohanty, 2016). Particularmente, el modelo que prueba que el compromiso afectivo media parcialmente las relaciones entre las dimensiones de la articulación trabajo-familia (CTF y ETF) y la satisfacción laboral resulta totalmente novedoso por las particularidades ya expuestas. El mismo puede ser explicado dado que en el proceso de formación de estos lazos afectivos interviene la valoración que el empleado realiza del apoyo y los beneficios recibidos por parte de la organización. Si tales evaluaciones resultan positivas, el trabajador percibirá los objetivos de la empresa como si fueran propios y deseará seguir perteneciendo a la misma porque la considera parte de su vida (Omar \& Urteaga, 2008). En este sentido, es probable que el empleado intercambie su compromiso por el acceso a determinadas políticas amigables con la familia que conllevan la posibilidad de balancear las demandas provenientes de los roles familiares y laborales. Estos niveles de compromiso afectivo, a su vez, podrían derivar en un mayor grado de satisfacción laboral (Cropanzano, Anthony, Daniels, \& Hall, 2017). En concordancia, la teoría del estrés de rol sostiene que los empleados altamente comprometidos con su organización tienden a generar sentimientos de estabilidad y de seguridad para con la empresa que le permiten hacer frente a los conflictos derivados de la dificultad de articular las demandas de ambas esferas (Buonocore \& Russo, 2013).

Los resultados aquí obtenidos podrían ser de utilidad para gerentes, líderes o personas que se encuentren relacionadas a la gestión de los recursos humanos a la hora de evaluar la implementación de políticas de trabajo flexible amigables con la familia dado su impacto sobre aspectos tales como la retención del talento (compromiso) y la percepción que estos tienen sobre sus empleos. Asimismo, el hecho de mantener altamente motivados (satisfechos) a los trabajadores del sector terciario al momento de realizar las tareas propias del rol se traslada esa actitud positiva a los clientes, que aportan a la rentabilidad de la organización. En este tipo de organizaciones, una buena atención puede lograr competitividad a la hora de buscar y retener a los consumidores. Es por ello que el capital humano se ha convertido en una clave para el éxito de las compañías de servicios o comercios (Guerrero, Parra, \& Arce, 2018). 
Como toda investigación empírica, la presente no se encuentra exenta de ciertas debilidades y fortalezas. En lo que respecta a las limitaciones, vale aclarar que si bien el estudio se diseñó teniendo en cuenta una estrategia asociativa-explicativa (Ato et al., 2013), el mismo se orienta más hacia una investigación de carácter exploratorio, dado que desde un principio: (a) no se propone exclusivamente a un tipo de compromiso organizacional como el posible mediador, sino que la selección del compromiso afectivo (así como el título de la presente publicación) surge $a$ posteriori de los análisis preliminares, exploratorios, y no de una hipótesis previa; (b) ni se tuvo en cuenta el tipo de mediación (total o parcial) que podría esperarse entre las variables de interés, la cual se determina a partir de la contrastación de dos modelos rivales. El carácter transversal de la investigación también podría considerarse una debilidad del estudio en tanto impide la realización de inferencias de causalidad entre las variables de interés. Al respecto, futuras investigaciones podrían emplear diseños longitudinales a los fines de establecer dicho orden causal. En tercer lugar, dado que el procedimiento utilizado para la selección de la muestra ha sido no probabilístico y se desconoce el número total de trabajadores pertenecientes a las organizaciones que participaron del estudio, los resultados aquí obtenidos no podrían ser generalizados al conjunto de la población objetivo. Para superar tal debilidad, estudios venideros deberían enriquecer el método de selección de participantes a través de la representatividad de la muestra y de valorar el posible sesgo de selección de los mismos. En este sentido, por ejemplo, podrían valerse de muestras aleatorias que permitan representar adecuadamente los diversos estratos ocupacionales y conglomerados geográficos. Cuarto, la prueba de factor único de Harman tiene sus limitaciones, por lo que en la evaluación de los posibles sesgos del método común se podría sumar la técnica de medición directa del factor latente (MacKenzie \& Podsakoff, 2012) para identificar la naturaleza del mismo. Una última limitación podría estar vinculada al carácter autodescriptivo de las escalas utilizadas las que, además, podrían ser proclives a la tendencia de los participantes a mostrar una imagen mejorada de sí mismos. No obstante, cabe aclarar que todos los trabajadores tuvieron garantizado el anonimato durante el proceso de recolección y análisis de los datos. En definitiva, esta debilidad podría sobrellevarse mediante la inclusión de escalas de deseabilidad social o datos provenientes de otras fuentes más objetivas.

Entre las fortalezas, esta investigación constituye un aporte original a la literatura psicológica desde el momento que (a) corrobora que el compromiso afectivo es la medida más efectiva del compromiso organizacional, (b) prueba el potencial predictivo de las dimensiones de la articulación trabajo-familia sobre la satisfacción laboral de manera conjunta, (c) verifica que el compromiso afectivo media parcialmente el vínculo entre dichas variables, y (e) ofrece sustento teórico para las interacciones empíricamente verificadas. Por lo tanto, los hallazgos obtenidos contribuyen a cubrir un área de vacancia desde el momento que la revisión de la literatura más actualizada no arroja ningún estudio que haya examinado simultáneamente esta particular combinación de constructos.

Con base en estos resultados, futuros estudios podrían incorporar nuevas variables mediadoras que expliquen el efecto residual entre la satisfacción laboral y los aspectos de la articulación trabajofamilia. En este sentido, otras variables disposicionales (como las estrategias de afrontamiento del estrés) u organizacionales (como la autonomía laboral o el trabajo flexible) podrían ocupar este rol. Paralelamente, se podrían comparar los resultados obtenidos con los de otros sectores económicos para verificar si cada rubro presenta particularidades en lo que hace al interjuego de las variables propuestas.

\section{Conflicto de intereses}

El trabajo enviado no presenta ningún conflicto de interés. 


\section{Responsabilidad ética}

Se tomaron en consideración todos los recaudos éticos vinculados con la investigación con seres humanos establecidos por la Asociación Americana de Psicología (2017) y las recomendaciones del CONICET (2006) para las investigaciones en las ciencias sociales y humanas.

\section{Contribución de autoría}

El trabajo ha sido realizado en su totalidad por el autor.

\section{Agradecimientos}

El presente trabajo ha sido realizado en el marco de la beca posdoctoral otorgada al autor por el CONICET (Consejo Nacional de Investigaciones Científicas y Técnicas), y dirigida por la Dra. Alicia Omar (CONICET; Universidad Nacional de Rosario, Argentina.

\section{Referencias}

Al Momani, H. M. (2017). The Mediating Effect of Organizational Commitment on the Relationship between Work-Life Balance and Intention to Leave: Evidence from Working Women in Jordan. International Business Research, 10(6), 164-177. doi: 10.5539/ibr.v10n6p164

AlAzzam, M., AbuAlRub, R. F., \& Nazzal, A. H. (2017). The Relationship between Work-Family Conflict and Job Satisfaction among Hospital Nurses. Nursing Forum, 52(4), 278-288. doi: 10.1111/nuf.12199

Albdour, A. A., \& Altarawneh, I. I. (2014). Employee Engagement and Organizational Commitment: Evidence from Jordan. International Journal of Business, 19(2), 192212. Recuperado de https://pdfs.semanticscholar.org/fd0f/ fc62aa4b60c4b4d695f510845bce7bca75ed.pdf

Allen, T. D., Johnson, R. C., Kiburz, K. M., \& Shockley, K. M. (2013). Work-Family Conflict and Flexible Work Arrangements: Deconstructing Flexibility. Personnel Psychology, 66(2), 345-376. doi: 10.1111/peps.12012

Allen, N. J., \& Meyer, J. P. (1990). The Measurement and Antecedents of Affective, Continuance and Normative
Commitment to the Organization. Journal of Occupational Psychology, 63(1), 1-18. doi: 10.1111/j.20 44-8325.1990.tb00506.x

American Psychological Association. (2017). Ethical principles of psychologists and code of conduct (2002, amended effective June 1, 2010, and January 1, 2017). Recuperado de http://www.apa.org/ethics/code/ index.html

Ato, M., López, J. J., \& Benavente, A. (2013). Un sistema de clasificación de los diseños de investigación en Psicología. Anales de Psicología, 29(3), 1038-1059. doi: 10.6018/analesps.29.3.178511

Bagherzadeh, R., Taghizadeh, Z., Mohammadi, E., Kazemnejad, A., Pourreza, A., \& Ebadi, A. (2016). Relationship of Work-Family Conflict with Burnout and Marital Satisfaction: Cross-Domain or Source Attribution Relations? Health Promotion Perspectives, 6(1), 31-36. doi: 10.15171/hpp.2016.05

Bakker, A. B., \& Demerouti, E. (2013). La teoría de las demandas y los recursos laborales. Revista de Psicología del Trabajo y de las Organizaciones, 29(3), 107-115. doi: 10.5093/tr2013a16

Baron, R. M., \& Kenny, D. A. (1986). The moderatormediator variable distinction in social psychological research: Conceptual, strategic, and statistical considerations. Journal of Personality and Social Psychology, 51(6), 1173-1182. doi:10.1037/0022-35 14.51.6.1173

Bentler, P. (2006). EQS 6 Structural Equations Program Manual. Encino, CA: Multivariate Software.

Brougham, D., Haar, J., \& Roche, M. (2015). Work-Family Enrichment, Collectivism, and Workplace Cultural Outcomes: A Study of New Zealand Maori. New Zealand Journal of Employment Relations, 40(1), 1934. Recuperado de https://hdl.handle.net/10289/9933

Brummelhuis, L. L. ten, \& Bakker, A. B. (2012). A Resource Perspective on the Work-Home Interface: The WorkHome Resources Model. American Psychologist, 67(7), 545-556. doi: 10.1037/a0027974

Buonocore, F., \& Russo, M. (2013). Reducing the Effects of Work-Family Conflict on Job Satisfaction: The Kind of Commitment Matters. Human Resource Management Journal, 23(1), 91-108. doi: 10.1111/j.1748-8583.2011.00187.x 
Chhabra, B., \& Mohanty, R. P. (2016). Moderating Role of Locus of Control in the Relationship between Job Satisfaction and Organisational Citizenship Behaviour: A Study of the Indian IT Sector. International Journal of Indian Culture and Business Management, 13(3), 288-309. Recuperado de https://EconPapers.repec.org/ RePEc:ids:ijicbm:v:13:y:2016:i:3:p:288-309

CONICET (2006). Lineamientos para el comportamiento ético en las Ciencias Sociales y Humanidade. Buenos Aires: CONICET.

Cooklin, A. R., Westrupp, E., Strazdins, L., Giallo, R., Martin, A., \& Nicholson, J. M. (2015). Mothers’ Work-Family Conflict and Enrichment: Associations with Parenting Quality and Couple Relationship. Child: Care, Health and Development, 41(2), 266-277. doi: 10.1111/cch.12137

Crain, T. L., \& Hammer, L. B. (2013). Work-Family Enrichment: A Systematic Review of Antecedents, Outcomes, and Mechanisms. In A. B. Bakker (Ed.), Advances in Positive Organizational Psychology (pp. 303-328). Howard House, UK: Emerald Group Publishing Limited.

Cropanzano, R., Anthony, E. L., Daniels, S. R., \& Hall, A. V. (2017). Social Exchange Theory: A Critical Review with Theoretical Remedies. Academy of Management Annals, 11(1), 479-516. doi: 10.5465/annals.2015.0099

Fazio, J., Gong, B., Sims, R., \& Yurova, Y. (2017). The Role of Affective Commitment in the Relationship between Social Support and Turnover Intention. Management Decision, 55(3), 512-525. doi: 10.1108/MD-05-2016-0338

Fornell, C., \& Larcker, D. F. (1981). Structural Equation Models with Unobservable Variables and Measurement Error: Algebra and Statistics. Journal of Marketing Research, 18(3), 382-388. doi: 10.2307/3150980

Frone, M. R. (2003). Work-Family Balance. In J. C. Quick \& L. E. Tetrick (Eds.), Handbook of occupational health psychology (pp. 143-162). Washington, DC: American Psychological Association.

Gabini, S. (2017). Interacción trabajo-familia: Adaptación y validación de un instrumento para medirla. Revista da UIIPS, 5(5), 24-32. Recuperado de http:// lms.essaude.ipsantarem.pt/index.php/REVUIIPS/article/ view/438
Geurts, S. A., Taris, T. W., Kompier, M. A., Dikkers, J. S., Hooff, M. L. van, \& Kinnunen, U. M. (2005). WorkHome Interaction from a Work Psychological Perspective: Development and Validation of a New Questionnaire, the SWING. Work \& Stress, 19(4), 319339. doi: 10.1080/02678370500410208

Greenhaus, J. H., \& Powell, G. N. (2006). When Work and Family are Allies: A Theory of Work-Family Enrichment. Academy of Management Review, 31(1), 72-92. doi: 10.5465/amr.2006.19379625

Guerrero, M. A., Parra, R. J., \& Arce, M. F. (2018). La satisfacción laboral y su efecto en la satisfacción del cliente, un análisis teórico. INNOVA Research Journal, 3(8), 140-146. doi: 10.33890/innova.v3.n8.2018.879

Haar, J. M., Russo, M., Suñe, A., \& Ollier-Malaterre, A. (2014). Outcomes of Work-Life Balance on Job Satisfaction, Life Satisfaction and mental health: A study across seven cultures. Journal of Vocational Behavior, 85(3), 361-373. doi: 10.1016/j.jvb.2014.08.010

Hair, J. F., Black, W. C., Babin, B. J., Anderson, R. E., \& Tatham R. L. (2010). Multivariate Data Analysis (6 ${ }^{a}$ ed.). Upper Saddle River, NJ: Pearson-Prentice Hall.

Hopwood, C. (2007). Moderation and Mediation in Structural Equation Modeling: Applications for Early Intervention Research. Journal of Early Intervention, 29(3), 262-272. doi: 10.1177/105381510702900305

Jijena-Michel, R. D., \& Jijena-Michel, C. E. (2015). El rol moderador de la flexibilidad del horario de trabajo en la relación del enriquecimiento trabajo familia y la satisfacción docente. Horizontes Empresariales, 10(2), 41-56. Recuperado de http://revistas.ubiobio.cl/inde x.php/HHEE/article/view/2018

Judge, T. A., \& Kammeyer-Mueller, J. D. (2012). General and Specific Measures in Organizational Behavior Research: Considerations, Examples, and Recommendations for Researchers. Journal of Organizational Behavior, 33(2), 161-174. doi: 10.1002/job.764

Kaur, H., \& Soch, H. (2018). Satisfaction, Trust and Loyalty: Investigating the Mediating Effects of Commitment, Switching Costs and Corporate Image. Journal of Asia Business Studies, 12(4), 361-380. doi: 10.1108/JABS-082015-0119 
Leggat, S. G., Karimi, L., \& Bartram, T. (2017). A Path Analysis Study of Factors Influencing Hospital Staff Perceptions of Quality of Care Factors Associated with Patient Satisfaction and Patient Experience. BMC Health Services Research, 17(1), 39-53. doi: 10.1186/s12913-0172718-x

Leineweber, C., Baltzer, M., Hanson, L. L. M., \& Westerlund, H. (2013). Work-Family Conflict and Health in Swedish Working Women and Men: A 2-Year Prospective Analysis. The European Journal of Public Health, 23(4), 710-716. doi: 10.1093/eurpub/cks064

Lingard, H. C., Francis, V., \& Turner, M. (2010). Work-Family Enrichment in the Australian Construction Industry: Implications for Job Design. Construction Management and Economics, 28(5), 467-480. doi: 10.1080/01446 190903511268

Liu, X. P., \& Wang, Z. M. (2013). Perceived Risk and Organizational Commitment: The Moderating Role of Organizational Trust. Social Behavior and Personality: An International Journal, 41(2), 229-240. doi: 10.2224/ sbp.2013.41.2.229

MacDonald, S., \& Maclntyre, P. (1997). The Generic Job Satisfaction Scale: Scale Development and its Correlates. Employee Assistance Quarterly, 13(2), 1-16. doi: 10.1300/ J022v13n02_01

MacKenzie, S. B., \& Podsakoff, P. M. (2012). Common Method Bias in Marketing: Causes, Mechanisms, and Procedural Remedies. Journal of Retailing, 88(4), 542555. doi: 10.1016/j.jretai.2012.08.001

MacKinnon, D. P., Lockwood, C. M., Hoffman, J. M., West, S. G., \& Sheets, V. (2002). A comparison of methods to test mediation and other intervening variable effects. Psychological Methods, 7(1), 83-104. doi:10.1037/1082-989x.7.1.83

Marques, A. M., Chambel, M. J., \& Pinto, I. (2015). The Exchange Relationship between Work-Family Enrichment and Affective Commitment: The Moderating Role of Gender. The Spanish Journal of Psychology, 18, 1-11. doi: 10.1017/sjp.2015.38

Mauno, S., Cuyper, N. de, Kinnunen, U., Ruokolainen, M., Rantanen, J., \& Mäkikangas, A. (2015). The Prospective Effects of Work-Family Conflict and Enrichment on Job Exhaustion and Turnover Intentions: Comparing Long-
Term Temporary vs. Permanent Workers across Three Waves. Work \& Stress, 29(1), 75-94. doi: 10.1080/026783 73.2014.1003997

Mauno, S., \& Ruokolainen, M. (2017). Does Organizational Work-Family Support Benefit Temporary and Permanent Employees Equally in a Work-Family Conflict Situation in Relation to Job Satisfaction and Emotional Energy at Work and at Home? Journal of Family Issues, 38(1), 124148. doi: 10.1177/0192513X15600729

McNall, L. A., Scott, L. D., \& Nicklin, J. M. (2015). Do Positive Affectivity and Boundary Preferences Matter for WorkFamily Enrichment? A Study of Human Service Workers. Journal of Occupational Health Psychology, 20(1), 93104. doi: $10.1037 / \mathrm{a} 0038165$

Meyer, J. P., Stanley, D. J., Herscovitch, L., \& Topolnytsky, L. (2002). Affective, Continuance, and Normative Commitment to the Organization: A Meta-Analysis of Antecedents, Correlates, and Consequences. Journal of Vocational Behavior, 61(1), 20-52. doi: 10.1006/jvb e.2001.1842

Michel, J. S., \& Clark, M. A. (2009). Has it been Affect All Along? A Test of Work-to-Family and Family-to-Work Models of Conflict, Enrichment, and Satisfaction. Personality and Individual Differences, 47(3), 163-168. doi: 10.1016/j.paid.2009.02.015

Muneer, S., Iqbal, S., Khan, S., \& Long, C. S. (2014). An Incorporated Structure of Perceived Organizational Support, Knowledge-Sharing Behavior, Organizational Trust and Organizational Commitment: A Strategic Knowledge Management Approach. Pakistan Journal of Commerce and Social Sciences, 8(1), 42-57. Recuperado de http://www.jespk.net/publications/ 161.pdf

Oficina Económica y Comercial de España en Buenos Aires (2018). Informe económico y comercial. Argentina: Oficina Económica y Comercial de España en Buenos Aires. Recuperado de http://www.comercio.gob.es/tmp DocsCanalPais/C051C360DAC4A84922DEBEA5C90C F938.pdf

Omar, A., \& Urteaga, F. (2008). Valores personales y compromiso organizacional. Enseñanza e Investigación en Psicología, 13(2), 353-372. Recuperado de https:// www.redalyc.org/articulo.oa?id=292/29213210 
Omar, A., Urteaga, F., \& Salessi, S. (2015). Propiedades psicométricas de la Escala de Enriquecimiento TrabajoFamilia para la población argentina. Revista de Psicología, 24(2), 1-18. doi: 10.5354/0719-0581.2015.37689

Padhi, M., \& Pattnaik, S. (2013). A Typology of Work Family Research: Perspectives from Literature. Vilakshan: The XIMB Journal of Management, 10(2), 83-98.

Podsakoff, P. M., MacKenzie, S. B., \& Podsakoff, N. P. (2012). Sources of Method Bias in Social Science Research and Recommendations on How to Control it. Annual Review of Psychology, 63, 539-569. doi: 10.1146/annurev-psych120710-100452

Rahman, A., Sa’adah, N., Amin, S. M., Mahadi, N., \& Ismail, F. (2017). The Relationship between Work-Family Balance and Affective Organizational Commitment among Academic Staff of Malaysian Research Universities. Advanced Science Letters, 23(1), 482-485. doi: 10.1166/asl.2017.7229

Rantanen, J., Kinnunen, U., Mauno, S., \& Tement, S. (2013). Patterns of Conflict and Enrichment in Work-Family Balance: A Three-Dimensional Typology. Work \& Stress, 27(2), 141-163. doi: 10.1080/02678373.2013.791074

Rastogi, M., Rangnekar, S., \& Rastogi, R. (2016). Flexibility as a Predictor of Work-Family Enrichment. Global Journal of Flexible Systems Management, 17, 5-14. doi: 10.1007/s40171-015-0108-y

Romeo, M., Berger, R., Yepes-Baldó, M.., \& Ramos, B. (2014). Adaptation and Validation of the Spanish Version of the «Survey Work-Home Interaction-NijmeGen»(SWING) to Spanish Speaking Countries. Anales de Psicología, 30(1), 287-293. doi: 10.6018/analesps.30.1.148291

Salessi, S. (2014). Satisfacción laboral: acerca de su conceptualización, medición y estado actual del arte. Revista de Psicología, 10(19), 67-83. Recuperada de https://core.ac.uk/download/pdf/32622524.pdf

Salessi, S., \& Omar, A. (2016). Satisfacción Laboral Genérica. Propiedades psicométricas de una escala para medirla. Alternativas en Psicología, 20(34), 93108. Recuperado de http://hdl.handle.net/11336/53885

Salessi, S., \& Omar, A. (2017). Satisfacción laboral: un modelo explicativo basado en variables disposicionales.
Revista Colombiana de Psicología, 26(2), 329-345. doi: 10.15446/rcp.v26n2.60651.

Sangadji, E. M. (2014). The Effect of Organizational Culture on Lecturers' Job Satisfaction and Performance. International Journal of Learning and Development, 3(3), 1-18. doi: 10.5296/ijld.v3i3.3673

Scott, K. L., Ingram, A., Zagenczyk, T. J., \& Shoss, M. K. (2015). Work-Family Conflict and Social Undermining Behaviour: An Examination of PO Fit and Gender Differences. Journal of Occupational and Organizational Psychology, 88(1), 203-218. doi: 10.1111/ joop.12091

Simintiras, A., Watkins, A., Ifie, K., \& Georgakas, K. (2012). Individual and Contextual Influences on the Affective Commitment of Retail Salespeople. Journal of Marketing Management, 28(11-12), 1377-1398. doi: 10.1080/ 0267257X.2012.691529

Sobel, M. E. (1982). Asymptotic Confidence Intervals for Indirect Effects in Structural Equation Models. Sociological Methodology, 13, 290. doi:10.2307/270723

Tabachnick, B. G., \& Fidell, L. S (2013). Using Multivariate Statistics (6 $6^{\text {a }}$ ed.). Boston, MA: Pearson.

Timms, C., Brough, P., O’Driscoll, M., Kalliath, T., Siu, O. L., Sit, C., \& Lo, D. (2015). Positive Pathways to Engaging Workers: Work-Family Enrichment as a Predictor of Work Engagement. Asia Pacific Journal of Human Resources, 53(4), 490-510. doi: 10.1111/1744-7941.12066

Wayne, J. H., Randel, A. E., \& Stevens, J. (2006). The Role of Identity and Work-Family Support in Work-Family Enrichment and its Work-Related Consequences. Journal of Vocational Behavior, 69(3), 445-461. doi: 10.1016/j.jvb.2006.07.002

Wong, Y. W., \& Wong, Y. T. (2017). The Effects of Perceived Organizational Support and Affective Commitment on Turnover Intention. Journal of Chinese Human Resource Management, 8(1), 2-21. doi: 10.1108/JCHRM01-2017-0001

Yousef, D. A. (2017). Organizational Commitment, Job Satisfaction and Attitudes toward Organizational Change: A Study in the Local Government. International Journal of Public Administration, 40(1), 77-88. doi: 10.1080/01900692.2015.1072217 


\section{Sebastián Gabini}

Instituto de Investigaciones, Facultad de Humanidades y Artes, Universidad Nacional de Rosario, CONICET, Argentina.

Facultad de Psicología y Relaciones Humanas, Universidad Abierta Interamericana, Argentina.

Licenciado en Psicología (Universidad Abierta Interamericana), profesor universitario para la Educación Secundaria y Superior (Universidad Abierta Interamericana) y doctor en Psicología (Universidad Nacional de la Plata). Becario posdoctoral del CONICET con lugar de trabajo en el Instituto de Investigaciones de la Facultad de Humanidades y Artes, Universidad Nacional de Rosario (Argentina). Profesor asociado e investigador de la Facultad de Psicología y Relaciones Humanas, Universidad Abierta Interamericana (Argentina).

ORCID: https://orcid.org/0000-0002-0997-1423

Autor corresponsal: sebastian_gabini@live.com.ar 